\title{
Editorial: special issue on computational intelligence for vision and robotics
}

\author{
Jose Garcia-Rodriguez ${ }^{1} \cdot$ Isabelle Guyon $^{2} \cdot$ Sergio Escalera $^{3} \cdot$ Alexandra Psarrou $^{4}$. \\ Andrew Lewis ${ }^{5}$ Miguel Cazorla ${ }^{1}$
}

Published online: 26 April 2016

(C) The Natural Computing Applications Forum 2016

The International Joint Conference on Neural Networks (IJCNN) is the premier professional conference in the field of neural networks. A special session on Computational Intelligence for Vision and Robotics (CIVR) was organized in 2015 with a large volume of high-quality papers. A small number of outstanding papers presented at this special session were invited to submit extended versions of their work to this special issue. Additionally, a number of external papers were solicited, and only a selection of them was accepted after a rigorous revision process.

Over the last decades, there has been an increasing interest in robotics combined with computer vision techniques, to create autonomous systems that solve problems in different fields. This special issue aims to bring together research work in the area of computational intelligence applied to the fields of computer vision and robotics, investigating novel solutions and discussing the future trends in this field. Each paper was reviewed by at least two reviewers based on the preferences expressed by the Program Committee members, with at least two rounds before acceptance.

This compilation is composed of 8 accepted papers selected from 15 submissions. The papers are mainly related to neural representations of objects and/or scenes that improve classification tasks or present tools to test/

Jose Garcia-Rodriguez

jgarcia@dtic.ua.es

1 University of Alicante, Alicante, Spain

2 Clopinet, Berkeley, CA, USA

3 University of Barcelone, Barcelone, Spain

4 University of Westminster, London, UK

5 Griffith University, Brisbane, Australia improve computational intelligence methods applied to robotic vision problems. Clarke et al. present a real-time onboard autonomous object detection of moving objects from videos stream taken from a camera mounted to an unmanned aerial vehicle (UAV). Almomani et al. propose a novel Dirichlet process-based appearance model (DPAM) for object tracking modeling model the negative and positive target instances as the combination of multiple appearance models. Rekabdar et al. propose a novel unsupervised learning approach for spatiotemporal pattern classification. The authors use a spike-timing neural network with axonal conductance delays to learn the structure of spatiotemporal patterns from a small set of training samples. The network is then used for classifying unseen patterns. Elizondo et al. present a novel neural networkbased approach to recovering light source direction in relation to the viewpoint direction of a graphical image in noisy environments. Lopéz-Rubio et al. propose a self-organizing spanning tree model, which is able to learn a tree topology without any pre-specified structure. They applied the model to real video data for a foreground detection task. Escalante et al. propose an evolutionary algorithm capable of automatically learning weighting schemes based on bag-of-visual-words models for computer vision problems. García-García et al. present a new dataset for 3D object recognition using different RGB-D cameras and providing $3 \mathrm{D}$ pose information about objects in the scene, per-pixel segmentation and level of occlusion. In addition, it provides semiautomatic segmentation and semantic labels about the different parts of the objects so that the dataset could be used for testing robot grasping and scene labeling systems as well as for object recognition. SavalCalvo et al. present a comparative study of different downsampling techniques. They evaluate the effect of the downsampling in real applications like 3D non-rigid 
registration. From the results, they provide recommendations of kernels to apply in the sampling methods that drastically improve the results.
In summary, the selected papers present recent developments of computational intelligence-related research, with a focus on mobile robotics and computer vision. 